\title{
Central Venous Catheterization by Anesthesia Providers During Laparoscopic Radical Rectal Cancer Surgery: Unnecessary for Most Patients
}

\section{Zhenhua Lin}

The First Affiliated Hospital of Shantou University Medical College

Minghua Cheng

The First Affiliated Hospital of Shantou University Medical College

\section{Xiaoe Huang}

The First Affiliated Hospital of Shantou University Medical College

Liping Li ( $\square$ Ipli@stu.edu.cn )

Shantou University Medical College

\section{Research Article}

Keywords: central venous catheters, rectal cancer, unnecessary

Posted Date: March 12th, 2021

DOI: https://doi.org/10.21203/rs.3.rs-283554/v1

License: (c) (i) This work is licensed under a Creative Commons Attribution 4.0 International License. Read Full License 


\section{Abstract}

Background: Central venous catheter (CVC) is commonly used in surgery. The purpose of this retrospective study is to investigate whether there is an unnecessary use of CVC in laparoscopic radical rectal cancer.

Methods: This is a retrospective review of prospective medical records of patients who underwent laparoscopic radical rectal cancer surgery from January 2015 to May 2019 in our institution. Patients were divided into two groups according to whether CVC was inserted by the anesthesiologist or not, and the perioperative variables of the two groups were compared and analyzed. Patients with CVC insertion were divided into two groups according to their single-lumen or double-lumen, and the perioperative variables of the two groups were compared.

Results: Of 344 patients who underwent surgery, 194(56.4\%) were without CVC and 150 (43.6\%) with $\mathrm{CVC}$. There was no significant difference in operative time, fluid transfusion, blood loss, postoperative parenteral nutrition, and hospital stay $(p>0.05)$. For patients with CVC, none of the patients underwent central venous pressure monitoring, only $2(1.33 \%)$ patients received a blood transfusion, $11(7.33 \%)$ patients received vasoactive drugs and $3(2 \%)$ patients received postoperative ICU. There was no significant difference in perioperative variables between the single-lumen tube and double-lumen tube groups.

Conclusion: For most patients undergoing laparoscopic radical resection of rectal cancer, CVC is unnecessary and a waste of medical resources.

\section{Background}

Central venous catheter (CVC) is commonly applied in critically ill patients. CVC indications include the need for venous access, rapid fluid resuscitation, centrally administered intravenous therapies, central venous pressure monitoring, and hemodialysis[1,2]. Anesthesiologists preferred to expand CVC insertion indications in unforeseen circumstances for patients who undergo significant and prolonged surgery. However, CVC insertion is associated with significant risks, including catheter-related bloodstream infections, mechanical injuries, and venous thromboembolism.

Colorectal cancer (CRC), the second most common malignancy in the United States, seriously affects people worldwide[3]. Laparoscopic radical resection of rectal cancer has been widely accepted[4, 5]. Compared to traditional open approaches for colon cancer's surgical treatment, patients experience the same benefits of early recovery and decreased postoperative pain from the laparoscopic approach. Many anesthesiologists will insert CVC for patients undergoing laparoscopic radical rectal cancer surgery. However, not every surgical patient requires a CVC. Implanting unnecessary CVC for patients is undoubtedly harmful. It not only increases the risk of related-complications but also causes a waste of medical resources. For Patients with low-risk surgeries, less blood loss, short durations of anesthesia, and 
without postoperative intensive care and long-term parenteral nutrition after surgery, the application of CVC insertion in advance is unnecessary[6].

In China, the overuse of health care services has become an increasingly severe problem, increasing the burden of medical insurance and financial expenditure[7]. There were few studies on the overuse of central venous catheters during surgery. Based on this retrospective study, we attempted to explore the rationality of inserting $\mathrm{CVC}$ during laparoscopic radical resection of rectal cancer.

\section{Methods}

All methods were carried out in accordance with relevant guidelines and regulations.

The present study was approved by the Ethics Committee of the First Affiliated Hospital of Shantou University Medical College.

This study retrospectively analyzed data of patients who received laparoscopic radical rectal cancer surgery from January 2015 to May 2019 in our institution.

The study population were adult patients (age $\geq 18$ ) that underwent laparoscopic radical rectal cancer surgery from January 2015 to May 2019 in our institution. Exclusion criteria were active infection or sepsis and previous rectal surgery.

All patients received general anesthesia after the application of standard monitors. Anesthesiologists determine the methods of anesthesia induction and maintenance. After tracheal intubation, whether to indwelling CVC was concluded through discussion between anesthesiologist and surgeons. The anesthesiologist performed CVC placement. The location of the CVC placement depended on the operator's preference. The most common location was the right jugular vein.

Patients were divided into two groups according to whether CVC was inserted or not, and the perioperative variables of the two groups were compared and analyzed. Patients with CVC insertion were divided into two groups according to their single-lumen or double-lumen, and the perioperative variables of the two groups were compared.

Data were analyzed by IBM SPSS Statistical 26 , and the level of statistical significance was set at the conventional $p<0.05$. The quantitative data were analyzed using mean and standard deviation; the qualitative data were analyzed using frequency and percentage. Comparisons of quantitative data were performed using an independent-t-test categorical data were performed using the Pearson chi-square test.

\section{Results}

In total, 344 patients were included in this study. Table 1 shows the demographic characteristics of the study population. No significant differences were observed between the groups. Out of the 344 patients, 194(56.4\%) were without CVC and 150(43.6\%) with CVC. Out of the 150 patients with CVC, 55(36.66\%) were single-lumen, and 95(63.33\%) were double-lumen(Fig. 1). 
Table 1

Characteristics of the study population

\begin{tabular}{|c|c|c|c|}
\hline Item & No CVC & CVC & P-value \\
\hline Sample & $\mathrm{n}=194(56.40 \%)$ & $n=150(43.60 \%)$ & \\
\hline \multicolumn{4}{|l|}{ Gender } \\
\hline Male & $108(55.67 \%)$ & $86(57.33 \%)$ & \multirow[t]{2}{*}{0.76} \\
\hline Female & $86(44.33 \%)$ & $64(42.66 \%)$ & \\
\hline Age(yr) & $63.97 \pm 11.19$ & $63.83 \pm 10.95$ & 0.95 \\
\hline \multicolumn{4}{|c|}{ Surgical Methods } \\
\hline Dixion & 143(73.71\%) & 119(79.33\%) & \multirow[t]{3}{*}{0.22} \\
\hline Miles & $51(26.29 \%)$ & $30(20 \%)$ & \\
\hline Hartmann & 0 & $1(0.66 \%)$ & \\
\hline \multicolumn{4}{|l|}{ ASAPS } \\
\hline I & $10(5.15 \%)$ & $3(2 \%)$ & \multirow[t]{3}{*}{0.19} \\
\hline II & $171(88.14 \%)$ & $132(88 \%)$ & \\
\hline III & $13(6.70 \%)$ & $15(10 \%)$ & \\
\hline
\end{tabular}

Patients in the CVC group had a higher percentage of underlying diseases than those in the non-CVC group $(p=0.022)$. There were no statistically significant differences between the two groups in "Operative Time," "Fluid Transfusion," "Blood Loss," "Urine Output," "Blood Transfusion," "Vasopressor," "ICU," "Parenteral Nutrition," and "Postoperative Hospital Stay" (Table 2).

Out of 150 patients with CVC, none of the patients underwent central venous pressure monitoring, only 2 (1.33\%) patients received a blood transfusion, 11 (7.33\%) patients received vasoactive drugs, and 3 (2\%) patients received postoperative ICU. The blood loss of patients with CVC was only $600 \mathrm{ml}$ at most. Only $21(14 \%)$ received fluid transfusion volume over $3000 \mathrm{ml}$. 
Table 2

Comparison of perioperative variables between "CVC" and "No CVC" groups

\begin{tabular}{|c|c|c|c|}
\hline Item & No CVC & CVC & $P$-value \\
\hline \multicolumn{3}{|l|}{ Underlying Disease } & \multirow[t]{3}{*}{0.02} \\
\hline No & $107(55.15 \%)$ & $64(42.66 \%)$ & \\
\hline Yes & $87(44.84 \%)$ & $86(57.33 \%)$ & \\
\hline Operative Time(min) & $198.80 \pm 56.34$ & $206.30 \pm 58.86$ & 0.89 \\
\hline \multicolumn{4}{|l|}{ Fluid Transfusion(ml) } \\
\hline Total & $1875.52 \pm 434.98$ & $2200 \pm 467.03$ & 0.08 \\
\hline Crystalloids & $976.03 \pm 345.30$ & $1156.67 \pm 343.38$ & 0.02 \\
\hline Colloids & $902.06 \pm 323.03$ & $1046.67 \pm 354.01$ & 0.62 \\
\hline Blood Loss(ml) & $63.45 \pm 64.04$ & $69.97 \pm 102.52$ & 0.22 \\
\hline Urine Output(ml) & $405.26 \pm 277.37$ & $465.47 \pm 383.25$ & 0.15 \\
\hline \multicolumn{4}{|l|}{ Blood Transfusion } \\
\hline No & 193(99.48\%) & $148(98.66 \%)$ & \multirow[t]{2}{*}{0.42} \\
\hline Yes & $1(0.52 \%)$ & $2(1.33 \%)$ & \\
\hline \multicolumn{4}{|l|}{ Vasopressor } \\
\hline No & $188(96.91 \%)$ & 139(92.66\%) & \multirow[t]{2}{*}{0.07} \\
\hline Yes & $6(3.09 \%)$ & $11(7.33 \%)$ & \\
\hline \multicolumn{4}{|l|}{ ICU } \\
\hline No & 192(98.96\%) & 147(98\%) & \multirow[t]{2}{*}{0.46} \\
\hline Yes & $2(1.03 \%)$ & $3(2 \%)$ & \\
\hline \multicolumn{4}{|l|}{ Parenteral Nutrition } \\
\hline No & $4(2.06 \%)$ & $2(1.33 \%)$ & \multirow[t]{2}{*}{0.61} \\
\hline Yes & 190(97.94\%) & $148(98.66 \%)$ & \\
\hline Postoperative Hospital Stay(days) & $14.50 \pm 9.10$ & $13.90 \pm 8.70$ & 0.89 \\
\hline \multicolumn{4}{|c|}{$\begin{array}{l}\text { *Data was expressed as Mean } \pm \text { standard deviation or the number of patients. Parentheses indicate } \\
\text { percentage. }\end{array}$} \\
\hline \multicolumn{4}{|c|}{$\begin{array}{l}\text { Between the two groups with CVC, no significant differences were observed in "Age," "Underlying } \\
\text { Disease," "Fluid Transfusion," "Blood Loss," "Parenteral Nutrition," and "CVC Retention Time" (Table 3). } \\
\text { None of the } 150 \text { patients with CVC had related complications. }\end{array}$} \\
\hline
\end{tabular}


Table 3

Comparison of perioperative variables between "Single-lumen" and "double-lumen" groups

\begin{tabular}{|c|c|c|c|}
\hline \multirow[t]{2}{*}{ Item } & Single-lumen & Double-lumen & \multirow[t]{2}{*}{ P-value } \\
\hline & $n=55(36.66 \%)$ & $n=95(63.33 \%)$ & \\
\hline Age(yr) & $65.04 \pm 12.18$ & $63.13 \pm 10.17$ & 0.14 \\
\hline \multicolumn{4}{|l|}{ Underlying Disease } \\
\hline No & $26(47.27 \%)$ & $38(40 \%)$ & \multirow[t]{2}{*}{0.39} \\
\hline Yes & $29(52.72 \%)$ & $57(60 \%)$ & \\
\hline Fluid Transfusion(ml) & $2172.73 \pm 443.24$ & $2215.79 \pm 481.87$ & 0.26 \\
\hline Blood Loss(ml) & $69.45 \pm 103.02$ & $70.26 \pm 102.78$ & 0.99 \\
\hline Urine Output(ml) & $456.73 \pm 320.92$ & $470.53 \pm 416.64$ & 0.53 \\
\hline \multicolumn{4}{|l|}{ Parenteral Nutrition } \\
\hline No & 0 & $2(2.11 \%)$ & \multirow[t]{2}{*}{0.28} \\
\hline Yes & $55(100 \%)$ & $93(97.89 \%)$ & \\
\hline CVC Retention Time & $10.71 \pm 4.46$ & $10.37 \pm 7.68$ & 0.10 \\
\hline
\end{tabular}

\section{Discussion}

Central venous catheters (CVC) provide reliable venous access for various medical needs. With ultrasound positioning, central venous catheterization has become safer, more convenient, and faster[8, 9]. For significant and prolonged surgery, the anesthesiologist will often consider inserting a central venous catheter for the patient as appropriate to ensure stable venous access during the operation. In addition to fluid therapy and central venous pressure monitoring, it can also provide a stable channel for postoperative parenteral nutrition.

In response to an emergency during the operation, clinical anesthesiologists will appropriately relax the indications for central venous catheterization, which will lead to medical waste. In China, especially in some large medical units, anesthesiologists have a routine to indwell CVC on rectal cancer surgery patients.

However, small and short-term surgeries do not always require CVC. In low-risk surgeries and short anesthesia time, unnecessary CVC applications are increasing[6]. Compared with traditional open surgery, laparoscopic radical resection of rectal cancer has the advantages of less trauma, less bleeding, and 
shorter operation time and has been widely accepted[4]. The overutilization of central venous catheters can be found in this study.

The anesthesiologist might have overestimated the duration of the operation or the risk of the case. For most patients, intraoperative blood transfusion and application of vasoactive drugs are not required. In this study,Out of 150 patients with CVC, only $21(14 \%)$ received fluid transfusion volume over $3000 \mathrm{ml}$, and the blood loss of patients with CVC was only $600 \mathrm{ml}$ at most. CVC not only increases the risk of related complications but also wastes medical resources. Even if heavy bleeding during the operation or rapid fluid therapy is required, emergency inserting $\mathrm{CVC}$ is feasible.

The multi-lumen catheter has commonly used because of its advantages in treating patients who require a large amount of drug infusion. Bloodstream infections are the main complication of CVC. However, whether multi-lumen catheters increase the risk of infections is still controversial[10]. A meta-analysis suggests that multi-lumen catheters may be associated with a slightly higher risk of infections[11]. The application of antimicrobial-impregnated CVC[12] dramatically reduces the incidence of bloodstream infections. Thanks to the application of antimicrobial-impregnated CVC, none of the 150 patients with $\mathrm{CVC}$ had related complications. Although the risk of infection does not necessarily increase, using a multi-lumen catheter when a single-lumen catheter can meet the patient's infusion needs will undoubtedly increase vascular damage and medical costs.

Therefore, a sufficient assessment of the patient's operation time and blood loss before surgery can reduce unnecessary use of CVC as much as possible and reduce the patient's trauma and medical expenses.

Some surgeons believe that inserting CVC in advance can provide reliable venous access for parenteral nutrition while reducing peripheral vascular puncture and infusion. With advances in technical catheters, non-invasive hemodynamic monitoring and improved venipuncture techniques have also reduced the need for perioperative central venipuncture[13]. For patients with no peripheral blood vessel puncture obstacles and undergo short-term parenteral nutrition, peripheral parenteral nutrition can fully meet the needs[14].

Whether patients undergoing laparoscopic radical resection of rectal cancer should be routine parenteral nutrition is currently inconclusive, $[15,16]$ early enteral nutrition is more conducive to patients' recovery. Enhanced Recovery After Surgery (ERAS )[17] has been proven to reduce morbidity rates, improve recovery and shorten the length of stay in elective colorectal surgery. Resumption of oral nutrition on the first day after surgery after colorectal cancer surgery may be associated with improved 5-year cancerspecific survival[18]. The surgeon will insert peripherally inserted central catheters (PICCs) or infusion port for patients who need chemotherapy after surgery. Then the CVC inserted during the operation is a bit redundant.

Medical waste is increasingly recognized as the cause of patient harm and high costs. "Unnecessary services" is the most significant cause of waste in healthcare in the United States. In 2010, the Institute of 
Medicine called attention to this problem[19]. A United States survey on over-treatment found that the most common cause of over-treatment is "fear of malpractice" (84.7\%)[20], 71\% of respondents said they were more likely to perform unnecessary procedures when they profit from them.

This study had several limitations. First, this study was conducted in a single-center, resulting in the patients' selection bias. Second, the study was retrospective, and observational character relied on the quality and availability of data in the patient medical records. We cannot know whether the patient has difficulty in peripheral intravenous infusion before CVC catheter insertion, nor can we know the actual application of CVC catheter after surgery. None of the included patients had CVC catheter-related complications after surgery. However, it was impossible to see the patient's CVC insertion's specific process, including the number of punctures and whether mechanical complications occurred. It is necessary to develop guidelines for intraoperative inserting CVC through future prospective randomized controlled studies.

\section{Conclusions}

Preoperative insertion of central venous catheters by anesthesia providers during laparoscopic radical rectal cancer surgery is unnecessary for most patients. Whether to insert CVC should be more carefully considered according to patient's conditions and intraoperative conditions to avoid unnecessary injury and medical waste.

\section{Abbreviations}

ASAPS:American Society of Anesthesiologists physical status; CVC:central venous catheter; ERAS:Enhanced Recovery After Surgery; ICU:Intensive Care Unit; PICC:peripherally inserted central catheters

\section{Declarations}

\section{Declaration of interest}

The authors declare no conflicts of interest.

\section{Acknowledgments}

Not applicable.

\section{Authors' contributions}

ZL participated in the design of the study, performed the study, helped with the statistical analysis, and drafted the manuscript. MC and XH performed the data collection. LL were in charge of the conception 
and design the manuscript. All authors read and approved the final manuscript.

\section{Funding}

There was no funding source in this study.

\section{Availability of data and materials}

All data generated or analyzed during this study are included in this published article. Raw data are available upon reasonable request from the corresponding author.

\section{Ethics approved and consent to participate}

The present study was approved by the Ethics Committee of the First Affiliated Hospital of Shantou University Medical College.(Code: B-2021-006), and written informed consent for this retrospective observational study was waived.

\section{Consent for publication}

Not applicable.

\section{Competing interests}

The authors declare that they have no competing interests.

\section{Author details}

${ }^{1}$ Department of Anesthesiology, The First Affiliated Hospital of Shantou University Medical College, No. 57, Changping Road, Jinping District, Shantou, Guangdong, China. ${ }^{2}$ Department of Ultrasound, The First Affiliated Hospital of Shantou University Medical College, No. 57, Changping Road, Jinping District, Shantou, Guangdong, China. ${ }^{3}$ Shantou University Medical College, 22 Xin Ling Road, Shantou 515041, China

\section{References}

1. Jamshidi R: Central venous catheters: Indications, techniques, and complications. Seminars in Pediatric Surgery 2019. 
2. Bonnie F, Marianna S: Central Venous Catheter. American journal of respiratory and critical care medicine 2019, 199(11).

3. Siegel RL, Miller KD, Goding Sauer A, Fedewa SA, Butterly LF, Anderson JC, Cercek A, Smith RA, Jemal A: Colorectal cancer statistics, 2020. CA: A Cancer Journal for Clinicians 2020.

4. Vennix S, Pelzers L, Bouvy N, Beets GL, Pierie JP, Wiggers T, Breukink S: Laparoscopic versus open total mesorectal excision for rectal cancer. Cochrane Database of Systematic Reviews 2014.

5. Martijn Hgm vdP: Laparoscopic versus open surgery for rectal cancer (COLOR II): short-term outcomes of a randomised, phase 3 trial. The Lancet Oncology 2013, 3(14).

6. Keiko U, Satoki I, Masahiko K: The unnecessary application of central venous catheterization in surgical patients. Brazilian Journal of Anesthesiology 2018, 68(4):336-343.

7. Zhang Y, Zhou Z, Si Y: When more is less: What explains the overuse of health care services in China? Social Science \& Medicine 2019, 232.

8. Saugel B, Scheeren TWL, Teboul JL: Ultrasound-guided central venous catheter placement: a structured review and recommendations for clinical practice. Critical care (London, England) 2017, 21(1):225.

9. Peris A, Zagli G, Bonizzoli M, Cianchi G, Ciapetti M, Spina R, Anichini V, Lapi F, Batacchi S: Implantation of 3951 long-term central venous catheters: performances, risk analysis, and patient comfort after ultrasound-guidance introduction. Anesthesia and analgesia 2010, 111(5):1194-1201.

10. Devin, Callister, Pauline, Limchaiyawat, Samantha J, Eells, Loren G, Miller: Risk factors for central line-associated bloodstream infections in the era of prevention bundles. Infection control and hospital epidemiology 2015.

11. Dezfulian C, Lavelle J, Nallamothu BK, Kaufman SR, Saint S: Rates of infection for single-lumen versus multilumen central venous catheters: a meta-analysis. Critical Care Medicine 2003, 31(9):2385-2390.

12. Lai NM, Chaiyakunapruk N, Lai NA, O'Riordan E, Saint S: Catheter impregnation, coating or bonding for reducing central venous catheter-related infections in adults. Cochrane Database of Systematic Reviews 2013, 6(6):CD007878.

13. Rubin DS, Apfelbaum JL, Tung A: Trends in Central Venous Catheter Insertions by Anesthesia Providers: An Analysis of the Medicare Physician Supplier Procedure Summary From 2007 to 2016. Anesthesia and analgesia 2020, 130(4):1026-1034.

14. Anderson ADG, Palmer D, Macfie J: Peripheral parenteral nutrition. Br J Surg 2010, 90(9).

15. Barneveld KWYV, Smeets BJJ, Heesakkers FFBM, Bosmans JWAM, Boelens PG: Beneficial Effects of Early Enteral Nutrition After Major Rectal Surgery: A Possible Role for Conditionally Essential Amino Acids? Results of a Randomized Clinical Trial. Critical Care Medicine 2016, 44(6):e353.

16. Boelens PG, Heesakkers FFBM, Luyer MDP, Van Barneveld KWY, De Hingh IHJT, Nieuwenhuijzen GAP, Roos AN, Rutten HJT: Reduction of postoperative ileus by early enteral nutrition in patients undergoing major rectal surgery: prospective, randomized, controlled trial. Annals of Surgery 2014, 259(4):649. 
17. Gustafsson UO, Scott MJ, Hubner M, Nygren J, Ljungqvist O: Guidelines for Perioperative Care in Elective Colorectal Surgery: Enhanced Recovery After Surgery (ERAS) Society Recommendations: 2018. World Journal of Surgery 2018, 43(787-795).

18. Gustafsson UO, Oppelstrup H, Thorell A, Nygren J, Ljungqvist $O$ : Adherence to the ERAS protocol is Associated with 5-Year Survival After Colorectal Cancer Surgery: A Retrospective Cohort Study. World Journal of Surgery 2016, 40(7):1741-1747.

19. Medicine IO: Best Care at Lower Cost: The Path to Continuously Learning Health Care in America. Patient Safety \& Quality Healthcare 2013, 67(6).

20. Heather L, Tim X, Daniel B, Brandan MB, Michol C, Michael D, Wick EC, Vikas S, Shannon B, Makary MA: Overtreatment in the United States. Plos One 2017, 12(9):e0181970.

\section{Figures}

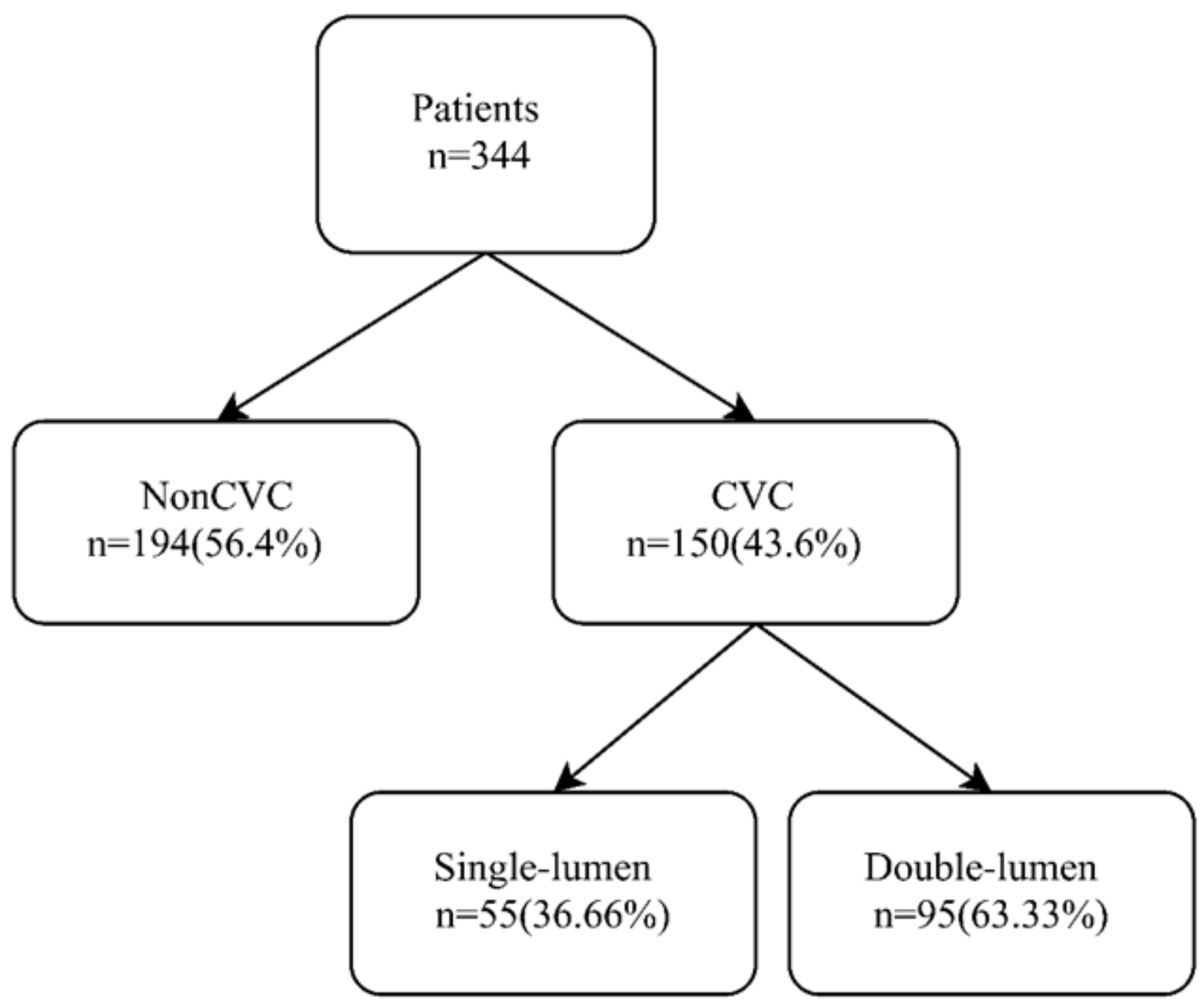

Figure 1 
Illustration of the data collection process. 